\title{
Degeneración y eugenesia en la historia de la siquiatría brasileña: Renato Kehl y los heredo-degenerados
}

\section{Degeneration and eugenics in the history of Brazilian psychiatry: Renato Kehl and hereditary degenerates}

Sandra Caponi

Professora, Departamento de Sociologia e Ciências Políticas/ Universidade Federal de Santa Catarina. Florianópolis - SC - Brasil

sandracaponi@gmail.com
CAPONI, Sandra. Degeneración y eugenesia en la historia de la siquiatría brasileña: Renato Kehl y los heredodegenerados. História, Ciências, Saúde - Manguinhos, Rio de Janeiro, v.25, supl., ago. 2018, p.159-178.

\section{Resumen}

Se analiza de qué modo se articulan las nociones de degeneración y eugenesia en el campo de la siquiatría, en las primeras décadas del siglo XX, destacando la existencia de semejanzas y continuidades, pero también señalando sus diferencias y desacuerdos. Se analiza un momento particular de la historia de la siquiatría brasileña, en el cual los argumentos provenientes de la teoría de la degeneración y los argumentos e intervenciones eugenésicas se superponen y confunden, tomando como eje de discusión, entre otros textos, el trabajo de Renato Kehl denominado, "A esterilização dos grandes degenerados e criminosos".

Palabras clave: eugenesia; degeneración; biopolítica; siquiatría.

\section{Abstract}

This article analyzes the notions of degeneration and eugenics in the field of psychiatry in the first decades of the twentieth century, stressing the existence of similarities and continuities, but also differences and conflicts between them. It analyzes a particular moment in the history of Brazilian psychiatry when arguments from degeneration theory and eugenic arguments and interventions became superimposed and mingled. It focuses, among other texts, on the work of Renato Kehl, "A esterilização dos grandes degenerados e criminosos".

Keywords: eugenics; degeneration; biopolitics; psychiatry. 
$\mathrm{C}$ uando analizamos los trabajos dedicados a la defensa de argumentos e intervenciones eugenésicas que se multiplicaron en las primeras décadas del siglo XX, en el campo de la siquiatría, nos deparamos con una asociación que no deja de repetirse hasta llegar a parecer obvia o inevitable, al menos en Brasil y Argentina. Me refiero a la asociación insistentemente reiterada entre las nociones de "degeneración" y "eugenesia".

Será analizado aquí un momento particular de la siquiatría brasileña en el cual los argumentos provenientes de la teoría de la degeneración y los argumentos e intervenciones eugenésicas se superponen y confunden. Esta no es una exclusividad de la siquiatría brasileña, ni recubre la totalidad de la historia de la siquiatría de ese país. Tal como afirma Vanderlei de Souza, entre las décadas de 1920 y 1930, existieron importantes controversias en el campo de la siquiatría brasileña entre aquellos autores que, como Renato Kehl, defendían la eugenesia negativa y la esterilización de los enfermos mentales y degenerados, y autores que defendían una eugenesia preventiva (Stepan, 2005) y no aceptaban esas medidas radicales, como Roquette-Pinto (Sousa, 2016, p.93). Se trata aquí de analizar los argumentos presentados por el primero de estos dos grupos, deteniéndonos, en un segundo momento, en la lectura de algunos escritos puntuales de Renato Kehl. En esos escritos, Kehl reproduce la reiterada asociación entre eugenesia y degeneración, vinculando esas nociones a la defensa de intervenciones siquiátricas más radicales, conservadoras y autoritarias, centradas en la idea de la esterilización eugénica de los heredo-degenerados.

Inicialmente, se analizan las nociones de degeneración y eugenesia, y el modo como las mismas pudieron articularse en el campo de la siquiatría de inicios del siglo XX. Posteriormente, será analizado de qué modo esa asociación es retomada en los argumentos construidos por Renato Kehl en torno a la problemática de la locura. Necesariamente deberemos hacer un recorte en la inmensa producción científica de este prolífico autor, que cuenta con más de veinte libros publicados entre los años 1920 y 1940, además de una enorme cantidad de artículos dedicados a divulgar sus ideas eugénicas. De la totalidad de esa producción, minuciosamente discutida por varios autores (Pietta, Carvalho, 2016; Rolim, Sá, 2013; Souza, 2006a, 2006b, 2006c; Boarini, 2012), serán analizados aquí solo algunos textos. Particularmente, se analiza el libro Lições de eugenia, publicado en 1929, por ser considerado su trabajo más relevante, además de otros textos breves publicados en el Boletim de Eugenia y en los Arquivos Brasileiros de Higiene Mental, donde aparecen articuladas las nociones de degeneración, eugenesia y locura.

Ciertamente, la asociación entre esas nociones no es exclusiva de la siquiatría brasileña, ni se restringe a los textos de Renato Kehl aquí analizados. En las primeras décadas del siglo XX, podemos encontrar argumentos idénticos en la siquiatría alemana, en los argumentos de eugenistas norte americanos, como Charles Davenport (1911), o en la siquiatría de países de América Latina, como Argentina o Colombia, donde se reiteran acaloradas defensas de medidas de eugenesia negativa tales como la esterilización eugénica de los llamados heredo-degenerados.

Aunque la asociación entre las nociones de degeneración y eugenesia pueda parecer necesaria, ya que aparecen reiteradamente vinculadas, cuando nos detenemos a analizar los argumentos y las intervenciones propuestas aparecen significativas diferencias. Estas diferencias aparecen aun cuando, en ambos casos, la idea de herencia mórbida ocupe 
un lugar central. En L'Heredité morbide, Apert (1919, p.260) dirá que después de Magnan "la concepción de degeneración ha desbordado la medicina mental, siendo utilizada en criminología (Lombroso y su escuela) y en crítica literaria (Max Nardau), desde entonces el término degenerado se ha generalizado de tal modo que... los alienistas ya no reconocen a su hijo". 1

Apert, considera que ya no es posible hablar de herencia mórbida utilizando como referencia esa doctrina revestida de connotaciones ambiguas e ideas confusas. Afirmando que en el momento en que la degeneración está siendo abandonada como marco de referencia, Galton (1988), con sus estudios sobre herencia, abrió una nueva vía (Apert, 1919, p.263).

Quizás sea por estas y otras críticas semejantes que, en los Annales d'Hygiène Publique et de Médecine Légal y en los Annales Médico-psycologiques, es posible observar que las referencias a la degeneración en el campo de la siquiatría comienzan poco a poco a desaparecer. En los Annales d'Hygiène Publique et de Médecine Légal, la última referencia aparece en 1919. En el caso de los Annales Médico-psycologiques, las referencias se mantienen de forma cada vez menos frecuentes hasta 1930. En 1938 aparece una reseña del libro Degeneración y degenerados, del siquiatra argentino De Veyga. La noción de eugenesia, por otra parte, está prácticamente ausentes en los Annales d'Hygiène, habiendo tres textos dedicados a esa temática en los Annales Médico-psycologiques, uno escrito por un corresponsal de Filadelfia.

Será justamente en ese momento, cuando la teoría de la degeneración comienza a perder espacio, que las ideas de Galton se difunden en el mundo entero a partir de la creación de diversas sociedades de eugenesia, como la Sociedad Francesa de Eugenesia, en 1912, la Sociedad Eugénica Argentina, en 1918, y la Sociedad Eugénica Brasileña, en 1918, entre muchas otras.

\section{Degeneración y eugenesia}

Aun cuando la noción de degeneración parecía estar siendo abandonada por los siquiatras franceses, la asociación entre degeneración y eugenesia permanecerá inalterada, hasta la Segunda Guerra Mundial, en diferentes países del mundo. Así, un defensor de las leyes eugénicas como fue el siquiatra de Munich Ernest Rudin, colaborador de Emil Kraepelin en los estudios sobre esquizofrenia, se refiere insistentemente a las degeneraciones mentales. Rudin es más conocido por haber sido coautor de la ley de esterilización alemana, llamada Ley para la Prevención de Enfermedades Hereditarias, aprobada en 1933 por el régimen Nazi, aplicada en gran escala a los enfermos mentales en Alemania. Publica en 1937, un artículo en los Annales Médico-psycologiques, seguido de un debate, donde defiende la esterilización compulsiva de los degenerados:

Las enfermedades hereditarias no se pueden curar por tratamiento individual. Una vez que el carácter hereditario de ciertas enfermedades mentales está probado científicamente, por los métodos de investigación sobre gemelos, por el pronóstico de la herencia empírica, y por el estudio de árboles genealógicos, no resta para el eugenista, más que impedir la propagación de células sexuales 'tarée' con el método más humano de la esterilización y otras medidas eugénicas eliminatorias. Dejar la iniciativa de la 
esterilización a cargo de la decisión del enfermo o de sus representantes, significa renunciar a la raza en favor de la contra-selección (Rudin, 1937, p.370; énfasis en el original).

A la exposición de Rudin, le sigue un debate donde aparecen críticas de siquiatras de diferentes ciudades del mundo: Londres, Bolonia, Zurich, Limoge. Siendo la crítica más radical la del siquiatra francés Eugene Minkowski (1937, p.372), quien se declara abiertamente contrario a la esterilización obligatoria. Su crítica se refiere tanto a razones que hoy llamaríamos epistemológicas, por considerar que no existen elementos suficientes que permitan hablar de certezas en el campo de la herencia, como por razones políticas, en la medida en que Minkowski era un claro opositor al régimen Nazi y a la ley de protección encomendada a Rudin. Años más tarde, él y su familia sufrirán, como otros científicos franceses, la persecución y el odio irracional del Nazismo.

Minkowski (1937, p.371) se oponía, también, a toda forma de esterilización y a cualquier tipo de estrategia eugénica, defendiendo la idea de que "existe contradicción entre la tendencia liberal de la higiene mental y la tendencia autoritaria de la esterilización obligatoria", centrándose en la "capacidad de regeneración de los llamados degenerados".

Minkowski publica un año más tarde, en 1938, un extenso texto manifestando su preocupación por la ley de esterilización aprobada en Alemania, mostrando que la asociación entre degeneración y eugenesia no es necesaria, ni inmediata. Respondiendo, una vez más, a los argumentos políticos y seudocientíficos de Rudin, Minkowski (1938, p.482-183) afirma:

La ley de esterilización se arriesga a esterilizar, en primer lugar, al siquiatra y a la siquiatría, al aumentar singularmente la barrera entre este y el enfermo. Deja de lado toda la riqueza de hechos de la que debe ocuparse la siquiatría, al no interesarse más que por el cálculo esquemático del 'pronóstico hereditario' (Erbprognose). Negligencia así, más o menos conscientemente, los factores de regeneración progresiva que intervienen al lado de la degeneración. ... Por otra parte, del hecho de que la humanidad no haya sucumbido aún, en el curso de los siglos, a la degeneración progresiva, ¿no es una prueba de que las fuerzas reparadoras juegan constantemente?

A pesar de estas y otras críticas, la "ley de esterilización", tuvo un importante impacto en la siquiatría mundial, articulándose sin dificultad con los argumentos defendidos por los teóricos de la degeneración que, en las últimas décadas del siglo XIX e inicios del siglo XX, habían dejado un fuerte impacto en la siquiatría mundial. En ese momento, la teoría de la degeneración (Huertas, 2017) se transformó en un verdadero "programa de investigación" que concentró los esfuerzos de médicos, higienistas, juristas y siquiatras interesados en ampliar el alcance y los límites de la medicina mental (Coffin, 2003; Doron, 2016) que se difundirá por diferentes países del mundo: en Europa, aparece con fuerza en Italia, España y Alemania y en la siquiatría de América Latina, lo mismo ocurre en países como Colombia, México, Brasil y Argentina.

En el caso de Brasil, la noción de degeneración aparece insistentemente en el discurso de diversos siquiatras de la primera mitad del siglo XX, muchas veces asociado a la defensa de intervenciones eugénicas. Recordemos que año 1929 será realizado el primer Congreso Brasilero de Eugenesia, con la activa participación de Renato Kehl, quien ese 
mismo año publica Lições de eugenia. La lectura de artículos publicados entre 1929 y 1933 en el Boletim de Eugenia, dirigido por Renato Kehl, o de los trabajos publicados entre 1925 y 1947 en los Arquivos Brasileiros de Higiene Mental refuerzan la idea de que las propuestas eugénicas en el campo de la siquiatra estaban asociadas al problema de la degeneración.

\section{Los heredo-degenerados}

Quizás, la persistente asociación entre degeneración y eugenesia pueda ser mejor entendida si consideramos el impacto que la teoría de la degeneración había dejado en el campo de la salud mental y de la criminología en las últimas décadas del siglo XIX, en América Latina en general y en Brasil en particular.

A partir de la publicación del Tratado de degeneración física, moral y mental de Morel, en 1857, un número cada vez mayor de conductas pasaron a ser clasificadas como patologías siquiátricas. A partir del momento que la teoría de la degeneración se consolida como programa de investigación, la siquiatría pudo empezar a establecer vínculos directos entre un desvío de conducta y un estado anormal o patológico (heredado, pero definitivo).

Para comprender la teoría de la degeneración, es necesario hablar de un tipo particular de herencia, una herencia no definida, donde toda y cualquier anomalía puede surgir y multiplicarse a partir de un sujeto identificado como anormal. Esa herencia es denominada, siguiendo a Prosper Lucas, como "herencia disimilar". Foucault (1999) dirá que "el estudio de la herencia o la localización de la herencia en el origen de las anormalidades, constituye esa 'meta-somatización' que fue necesaria para construir el edificio de la degeneración". Los anormales no pueden ser considerados portadores de una enfermedad determinada, ellos anticipan un número ilimitado e indefinido de enfermedades posibles en su descendencia.

La degeneración se manifiesta, de manera progresivamente agravada, ya sea en las diversas generaciones de una misma familia o en las sucesivas etapas de vida de un mismo individuo, hasta llevar a un cuadro final irreversible. Morel (1857, p.15) enunciará el principio general de la degeneración, afirmando que "los seres degenerados forman grupos y familias con elementos distintivos relacionados invariablemente a las causas que los transformaron en lo que son: un desvío mórbido del tipo normal de la humanidad".

Morel introduce un tipo de explicación causal en la que se articulan factores internos (hereditarios) y externos. Se refiere a la ingestión de tóxicos, fundamentalmente alcohol, pero también a intoxicaciones provocadas por un medio patogénico, pantanos, suciedad, condiciones de trabajo. Partiendo de la herencia disimilar, afirma que una misma causa, ingestión de alcohol por ejemplo, puede provocar diversas patologías en los descendientes, tales como retardo mental, delirios, tendencia al crimen, prostitución etc. Estas ideas se divulgaron rápidamente en la comunidad de alienistas, a tal punto que Magnan y Legrain $(1895$, p.3) pudieron afirmar que "después de la aparición del tratado de degeneración de Morel, sus ideas impregnan casi todos los trabajos de patología mental, tanto en Francia como en el extranjero".

Pero será con Magnan que el concepto de degeneración ingresa como un saber legítimo en el campo de la siquiatría. Magnan presidió por más de diez años la Sociedad Médico-sicológica que reunía a todos los siquiatras franceses, desde 1850 hasta la segunda 
década del siglo XX. Defendía una clasificación de enfermedades mentales que integre a los heredo-degenerados. En el libro Les dégénérés (1895), Magnan y Legrain formulan algunas críticas a Morel, conservando, sin embargo, la idea de una natural tendencia de los degenerados graves hacia la esterilización natural. Así, la degeneración es definida por estos autores como:

El término degeneración aplicado a la patología mental designa el estado mórbido de un sujeto cuyas funciones cerebrales acusan un estado de imperfección notoria, si se lo compara con el estado cerebral de los tipos generadores. Este estado mórbido constitucional se agrava progresivamente, y así como la degeneración de un tejido precede a su desaparición, a su muerte, del mismo modo, la degeneración del individuo precede a la desaparición de la especie; la esterilidad es, en efecto, la marca última de la degeneración, ella precede a la destrucción del tipo. La degeneración es un estado patológico y no un estado regresivo, como lo comprenden ciertos autores (Magnan, Legrain, 1895, p.74).

Esta definición da continuidad a la idea defendida por Morel, según la cual los anormales llevaban inscripto en sus cuerpos su propia inviabilidad. "La herencia es vehículo de transmisión progresiva de toda forma de degeneración adquirida a lo largo de cuatro generaciones, hasta la esterilidad de esa última" (Serpa, 2006, p.128).

Para Magnan y Legrain (1895, p.64), existe un encadenamiento patológico en las degeneraciones mentales que lleva desde una predisposición mínima, con un leve desvío en relación a sus genitores hasta las formas más graves e irreversibles. De tal modo que

si observamos las enfermedades agrupadas en las dos primeras subdivisiones, puede observarse claramente que forman una cadena ininterrumpida desde el predispuesto mínimo, aquel que, por ejemplo, presenta un acceso único de melancolía simple en el curso de su vida, hasta el más tarado de los degenerados, el idiota, el imbécil, en el cual la ruina del sustrato mental es no solo un hecho consumado, sino que también la capacidad de reproducirse ha desaparecido.

A lo largo del libro Les dégénérés, los autores insisten en la importancia de crear estrategias de regeneración, como el control de las condiciones de vida, la alimentación, las condiciones de trabajo e higiene. Consideran que "ese debilitamiento se traduce por estigmas permanentes y esencialmente progresivos, salvo regeneración intercurrente. Cuando esta regeneración falta, se llegará, más o menos rápidamente, a la destrucción de la especie" (Magnan, Legrain, 1895 , p.80). Los autores, siguiendo a Morel, consideran que la esterilización no es un proceso ni inevitable ni deseable, sino que es en la regeneración intercurrente donde se juega la capacidad terapéutica de la psiquiatría.

Como ya fue dicho, la teoría de la degeneración se difundió rápidamente por diversos países de Europa y América Latina. Magnan, dirá que en Alemania será Krafft-Ebing (1903) quien dará a la degeneración y a la influencia hereditaria "su verdadero valor patológico" (Magnan, Legrain, 1895, p.31). Emil Kraepelin $(1907,1917)$ también retoma y tematiza el concepto de degeneración en diversos estudios. En 1908, publica un texto denominado On the question of degeneration (Kraepelin, 2007), donde reaparecen muchas de las premisas definidas por Morel y Magnan para clasificar las patologías mentales. 
Lo cierto es que, ni los degeneracionistas clásicos, como Morel y Magnan, ni los posteriores defensores de la teoría de la degeneración, como Kraepelin, se limitaron a definir clasificaciones confiables o medidas terapéuticas para los trastornos mentales. Ellos pretendían también crear intervenciones concretas en el espacio social, modificaciones en el medio que permitieran anticipar las patologías mentales, sea apostando a medidas de regeneración o a medidas de control eugenésico. Estrategias de intervención que, como veremos, podían adoptar perspectivas diferentes e incluso opuestas.

\section{Estrategias eugenésicas}

Las intervenciones sociales que Morel (1857) denomina "tratamiento moral", son diferentes y contrapuestas a las estrategias de intervención propuestas por Kraepelin (2007) en Alemania o por Renato Kehl (1929) en Brasil.

En los cincuenta años que separan al tratado de Morel del texto que, en 1908, Kraepelin dedica a la cuestión de la degeneración, el discurso eugenésico fue ganando terreno como un modo de controlar la amenaza representada por las enfermedades mentales y por la criminalidad. Como es sabido, diversas leyes eugénicas fueron promulgadas antes de la Segunda Guerra Mundial en diferentes países del mundo. Debemos recordar que ya en 1907 se había promulgado, en el estado americano de Indiana, la legislación que permitía la esterilización de los criminales y de enfermos mentales. En 1909 la misma legislación será aprobada para los estados de Washington, Connecticut y California hasta que en 1950 serán 33 los estados de América del Norte que poseen esa legislación. Lo mismo ocurrirá entre 1920 y 1935 en Suecia, Dinamarca, Finlandia, Noruega y Alemania (Pichot, 1995).

En 1857, Morel defendía como estrategias de regeneración y de prevención de las patologías mentales, intervenciones sociales higiénicas sobre el medio ambiente, tales como el control de las intoxicaciones provocadas por las condiciones insalubres de existencia, la mejoría de las condiciones de trabajo, el control de la miseria urbana. Kraepelin, por su parte, parece no compartir el optimismo de Morel. Para él, las estrategias de protección social a los individuos más vulnerables representaban un obstáculo para la purificación eugenésica de la raza. Para Kraepelin (2007, p.400):

En todo caso, es imposible saber cuántos idiotas, epilépticos, psicópatas, criminosos, meretrices y vagabundos son hijos de padres alcohólicos o sifilíticos y heredaron la inferioridad de sus padres. Es obvio que el daño será en parte compensado por su menor capacidad para sobrevivir. Pero, lamentablemente, la extensión de nuestros programas de asistencia social tiene el efecto de impedir a autopurificação natural de nuestro pueblo.

Es esta misma perspectiva la que encontramos en Brasil en los textos de Renato Kehl (1925, p.93), cuando afirma:

Se evidencia por todas partes la preocupación de los gobiernos por encontrar soluciones para abrigar y alimentar a un elevado porcentaje de incapaces, criminales y anormales. Para agravar la calamitosa situación, higiene, medicina y filantropía salvan 
la vida de millones de esos infra-hombres (que la selección natural debería eliminar) aumentando así las contribuciones para mantenerlos en inactividad o recluidos en instituciones.

Aunque exista una retórica darwiniana referida a "la lucha por la vida" o la "selección natural", con la que se pretende justificar el abandono social (Miranda, Vallejo, 2005), el argumento central que permite entender las articulaciones entre la teoría de la degeneración y los defensores de la eugenesia es la idea de una herencia mórbida que se agravaría en las sucesivas generaciones. Sin embargo, existen importantes diferencias en el modo como ambos tematizan la cuestión de la herencia. Mientras para degeneracionistas la idea de la herencia disimilar era la que permitía describir la sucesión de patologías existentes en las familias de los heredo-degenerados, para los Galtonianos eran las teorías de Mendel y de Weismann las que desempeñaban ese papel.

Si dejamos ahora de lado las referencias a intervenciones sociales más generales y nos restringimos al campo de la siquiatría, veremos que las diferencias permanecen. Así, en relación al control de matrimonios, Morel consideraba que los casamientos con personas sanas podían configurar estrategias de regeneración para enfermos mentales. Para Magnan y Legrain (1895, p.93) será preciso intervenir para impedir los casamientos, en el caso que ambos tengan estigmas de degeneración, aproximándose así a las tesis eugénicas y reforzando la idea de determinismo hereditario absoluto: "El degenerado es un enfermo en el origen ad ovo, se presenta con una tara constitucional que lo acompaña toda la vida de la cuna a la tumba, que debe pesar sobre la personalidad entera y debe hacer sentir su influencia en todas las etapas de la vida".

Pero es en el campo de la terapéutica siquiátrica, particularmente en lo que se refiere al tratamiento de los degenerados, donde aparecen, con mayor claridad, las diferencias con las tesis eugénicas defendidas, por ejemplo, por Renato Kehl $(1929,1925)$.

Para Magnan y Legrain, es prioritariamente en el medio familiar donde deben ser tratados los degenerados, debiendo ser excepcional la internación en caso de "paroxismo", esto es en caso de un ataque que represente alguna amenaza para sí o para los otros. Aunque podrá objetarse que difícilmente estas ideas hayan podido ser efectivamente realizadas, Magnan defiende una profilaxis no asilar, afirmando que se debe:

Aconsejar a esos enfermos a ir, en forma periódica, a tener una 'sesión' en el asilo, como otros toman un baño de mar. Muchos de los enfermos a los cuales estas prácticas fueron enseñadas se encuentran bien, cuando sienten sus ataques,ellos mismos saben que deben hacerse curar, y se imponen tratamientos temporarios en el asilo ... estas indicaciones se aplican a los sindrómicos. Cuando existen características peligrosas, el internamiento se impone como medida de seguridad. Pero aún en ese caso, el propio enfermo pide ayuda y considera a la internación como un beneficio (Magnan, Legrain, 1895, p.252).

Vemos aquí una propuesta de intervención diferente a la que surgirá a partir de 1908, cuando Francis Galton funda en Londres la Sociedad Eugenésica Británica. A partir de allí, y a lo largo de las primeras décadas del siglo XX, comenzará a ganar terreno y a fortalecerse un discurso que defiende intervenciones eugénicas radicales en el campo de la siquiatría: aislamiento, internación con fines eugenésicos y esterilización compulsiva. 
Renato Kehl (1929) resume en su libro Lições de eugenia la posición adoptada por el grupo de eugenistas brasileros que defendían la esterilización de enfermos mentales y criminales. En la Lección 11, dedicada a explicitar las medidas eugenésicas existe un apartado dedicado a la esterilización de los grandes degenerados y criminales, y otro dedicado a la cuestión del aborto por motivo eugenésico. Allí leemos: “Una medida propuesta por la eugenesia es la esterilización de los grandes degenerados y criminales. La simple interdicción legal de casamientos de estos individuos constituiría un 'medio atenuado', mientras que la esterilización representa un 'medio radical', muchas veces necesario (Kehl, 1929, p.199; énfasis en el original).

Renato Kehl se refiere también a lo que denomina de eugenesia preventiva, afirmando que cabe a la eugenesia propagar ideas regeneradoras, que permitan refirmar los caracteres óptimos transmisibles por herencia y, al mismo tiempo, eliminar las degeneraciones (Stepan, 2005). Sin embrago, y tal como afirman Robert Wegner y Vanderlei de Souza (2013), Kehl irá adoptando posiciones cada vez más escépticas en relación a la eugenesia preventiva y a la capacidad que la educación y la higiene realmente tendrían como instancias regeneradoras. De tal modo que para él: "hacer hombres buenos o malos", hacerlos "eugenicamente superiores", no dependería de la alimentación, del clima, de la religión y de la cultura, dependería antes de la "palanca principal del progreso biológico que es la aplicación de las leyes de la herencia, según los preceptos de la eugenesia" (Wegner, Souza, 2013, p.6).

En la lección mencionada, "Métodos y medios eugénésicos", destaca 13 medios básicos de intervención eugenésica. Los que aparecen en primer lugar son: (1) registro de pedigrees de familias, (2) segregación de deficientes, (3) esterilización de anormales y control de nacimientos. Dirá que "en este vasto programa se destaca lo que se refiere a la esterilización de individuos inaptos para las buenas procreaciones, esto es, los degenerados somatopsíquicos" (Kehl, 1929, p.220), insistiendo en el "valor eugenésico de la esterilización de degenerados".

Como es posible observar en estas referencias, en el caso de Brasil, al menos hasta la década de 1930, la asociación entre eugenesia y degeneración parecía firmemente establecida, independientemente de que existieran diferencias en las estrategias de intervención. Los eugenistas parecían desconsiderar las fuertes críticas que, desde el campo de la siquiatría, se dirigieron a la teoría de la degeneración. Muchas de ellas formuladas ya a fines del siglo XIX, como la enunciada por Séglas (1895, p.739) en sus Leçons cliniques sur les maladies mentales et nerveuses:

Solo el estudio de la embriología, de la patología, de la higiene, nos podrá ofrecer enseñamientos preliminares serios, constataciones absolutamente necesarias antes de concluir con un diagnóstico de degeneración, palabra vaga, de una elasticidad singular, que no hace más que esconder nuestra ignorancia de los hechos, hipótesis de una utilidad muy contestable cuando hoy se ha vuelto tan mal definida y sujeta a una cantidad de interpretaciones tan diferentes.

Críticas semejantes se multiplicaron en la primera década del siglo XX, muchas de ellas realizadas por seguidores de Galton, como Apert (1919), sin que eso implicara un obstáculo para que los eugenistas brasileros continuaran utilizando la noción de degeneración como marco explicativo general. 
Aun cuando las ideas de Galton sobre herencia, familias patológicas, desvío y normalidad estadística (Boarini, 2012) se construyeron independientemente de cualquier referencia a la teoría de la degeneración, el impacto que esta teoría ya había dejado en la siquiatría de diferentes partes del mundo posibilitó que cuestiones tales como la herencia mórbida y la eugenesia ingresaran, sin mayor dificultad, al campo de la siquiatría. Podemos decir que la teoría de la degeneración operó como condición histórica de posibilidad para el surgimiento, difusión y aceptación de las teorías eugenésicas defendidas en las primeras décadas del siglo XX.

La psiquiatría brasileña había integrado las ideas de los degeneracionistas, antes de la difusión de las ideas eugénicas, como puede observarse en el libro de Francisco Franco da Rocha (2008, p.151), Esboço de psiquiatría forense, publicado originariamente en 1904: “O maior número de casos difíceis, realmente, é fornecido pela degeneração nas suas múltiplas variedades - histeria, epilepsia, paranoia simples, loucura moral, obsessão e impulsão, dipsomania, prodigalidade, perversões sexuais etc. - mas outros casos ainda, fora da degeneração, apresentam dificuldades na aplicação das leis".

Degeneracionistas y eugenistas compartían un mismo punto de partida: el "mito de la normalidad hereditaria". Ambos querían identificar una serie de características físicas y comportamentales consideradas indeseables, por representar un desvío de la normalidad, para intervenir antes que esa patología se transforme en irreversible. Dos estrategias privilegiadas servirían para definir esas patologías: la identificación de estigmas y el uso de árboles genealógicos.

\section{La caza a los estigmas}

Aunque la "caza de los estigmas" (Carol, 1995) quedó inaugurada en 1857 con el tratado de Morel, a lo largo de la primera mitad del siglo XX, los estigmas no dejaron de multiplicarse, ampliándose el margen de intervención de siquiatras y criminólogos. En 1917, Kraepelin (1917, p.99) afirma que para reconocer patologías mentales hereditarias era necesario identificar "estigmas físicos, como malformaciones del paladar o de los órganos sexuales, estrabismo congénito, albinismo, posición errada de los dientes, de los ojos etc.". Las medidas del cráneo indicaban el desarrollo del córtex cerebral, y "un cuidadoso examen de las orejas podía revelar una causa suficiente para alucinaciones" (p.99).

Uno de los más reputados eugenistas franceses, miembro de la Sociedad Eugenésica de Francia y premio Nobel de Medicina, Charles Richet (1922), en un escrito denominado La selección humana, indica cuales eran los sujetos portadores de estigmas que debían ser considerados malos genitores, pasibles de interdicción y represión. Ese texto de Richet aparece diversas veces mencionado en el primer Congreso, particularmente en las conferencias dedicadas a la selección de casamientos, identificación de degenerados hereditarios y educación eugénica.

La lista de Richet presenta más de sesenta indicaciones: ser muy grande o muy pequeño; enfermo mental; contrahecho; alcohólicos; sifilíticos; epilépticos; degenerados; idiotas; muy gordos; malignos; rebeldes a toda disciplina; muy feos; criminosos; proxenetas etc. Para Richet (1922, p.150), "las virtudes que deben considerarse indispensables pueden 
ser resumidas en una palabra: la de normalidad. Es preciso que los progenitores no se desvíen de la medía general del tipo humano". Las condiciones exigidas para el progenitor ideal no estaban vinculadas a la idea de perfección, sino a la idea de normalidad como frecuencia. En la caracterización de la normalidad se transluce el ideal del justo medio: "ni muy grande ni mucho pequeño, el hombre no debe tener los cabellos muy lisos ni ensortijados, el vientre debe ser recto pero ligeramente redondeado, debe tener músculos, pero sin exageración" (p.151).

Richet será una de las referencias del trabajo de Joaquim Moreira da Fonseca (1929), "Casamiento y eugenesia", presentado en el primer Congreso Brasilero de Eugenia (RoquettePinto, 1929). El tipo medio como tipo normal, los cálculos de frecuencia, las curvas de Gauss de normalidad y desvío, así como las referencias al hombre medio de Quetelet, también aparecen diversas veces referenciados en ese Congreso con el objetivo de identificar normalidad y desvíos patológicos y de impedir la procreación de los anormales.

Renato Kehl realizó una minuciosa descripción de estigmas: la simetría o deformación de la cabeza, del rostro, de los miembros, afirmaron que los vicios de conformación de las orejas son indicadores de degeneración, así como el retardo intelectual y mental, el cretinismo, el alcoholismo, las enfermedades venéreas, y la criminalidad, todos ellos estigmas que llevarían a un proceso agravado de degeneración en los descendientes. Considera que no es posible partir, como Morel, de "un tipo medio ideal y universal" para definir estigmas. El tipo medio a ser considerado para fines comparativos es el hombre sano, alguien cuyos órganos están en perfecto funcionamiento, sin enfermedades o taras, cuyo cuerpo mantenga las proporciones correctas.

Entiende que la degeneración es un desvío de las cualidades de este individuo sano, debido a factores de orden hereditaria. Estos desvíos se hacen evidentes cuando observamos los estigmas de degeneración. "Nadie desconoce ciertos estigmas físicos indicativos de degeneración destacándose entre ellos la detención del desenvolvimiento, las aberraciones parciales o generales de la evolución, tales como la asimetría o deformación de la cabeza, del rostro, de los miembros, vicios de conformación de las orejas, entre otros" (Kehl, 1929, p.50).

Finalmente son tantos los signos indicadores de degeneración, que Kehl (1929, p.50) parece obligado a establecer algún límite, y lo encuentra en el lugar más inesperado cuando afirma que "no todo defecto físico puede ser entendido como estigma de degeneración. Por ejemplo, un individuo que pierde un brazo no es un degenerado ni ese defecto se transmite por herencia".

Este ejemplo nos da la pauta de la extensión ilimitada que podían alcanzar los estigmas de degeneración. Diez años antes, en 1919, Apert ya había advertido sobre la dificultad inherente a los estigmas de degeneración. Ellos podían ser indefinidamente ampliados, siempre podía definirse a un individuo como degenerado identificando alguna característica física o del carácter con un estigma.

No existe, por así decir, ninguna conformación física anormal que no haya sido considerada como estigma de degeneración. Por ir de la cabeza a los pies, señalemos la situación de los cabellos precozmente blancos, la baja implantación del cabello en la frente, su implantación en ángulo; la formación misma de la frente, elevada o chata, con la base frontal saliente, frente huidiza; la forma del cráneo, en torre, oblicuo, oval 
etc.; la forma de la nariz, con lóbulo adherente ... las irregularidades dentarias, las malformaciones cardiacas, la luxación de cadera, la obesidad, la pubertad precoz, la pubertad retardada, la incontinencia de orina (Apert, 1919, p.267).

Apert (1919, p.271) continua enumerando estigmas para concluir que "este número de ejemplos debería ser suficiente para mostrar hasta qué punto es necesario ser prudentes en la apreciación de los estigmas de degeneración".

La definición de estigma dada por Magnan y Legrain (1895, p.91) es amplia y ambigua: "Toda disposición orgánica, congénita y permanente cuyo efecto es obstaculizar el cumplimiento regular de la función correspondiente y destruir la armonía biológica, donde la especie encuentra de seguir su doble objetivo natural de conservación y reproducción". Esa definición debe complementarse con la enumeración de marcas o estigmas corporales que indiquen "un desequilibrio, una irregularidad, una asimetría" (p.91), a seguir será realizada una extensa enumeración de estigmas. Aunque esta enumeración confirma la pertinencia de la crítica de Apert (1919), una y otra vez veremos reaparecer referencias a estigmas físicos en los tratados de psiquiatría, criminología y eugenesia de la primera mitad del siglo XX.

\section{Sobre genealogías y esterilizaciones}

Otra estrategia común a los teóricos de la degeneración y a los eugenistas es el uso de árboles genealógicos. El estudio de Magnan (1893), Recherches sur les centres nerveux: alcoolisme, folie des héréditaires dégénérés, define un esquema que se repite, siendo la primera generación de alcohólicos simple; la segunda de alcohólicos con accidente cerebral; y la tercera de sujetos con accidentes convulsivos en la primera infancia. Muestra la evolución de familias de alcohólicos por tres o más generaciones, presentando invariablemente degeneraciones cada vez más graves.

Estas mismas genealogías se multiplican entre los eugenistas, con un objetivo biopolitico claro: la esterilización. Podemos hallar, aún en los años 1920 y 1930, genealogías semejantes presentadas en publicaciones científicas y en diarios de circulación masiva como modo de divulgar la importancia de la esterilización eugénica. En el Sunday Oregonian podemos leer un esquema simplificado extraído del libro de Richard Dugdale (1877) The Jukes: a study in crime, pauperism, diseases, and heredity. Ese esquema se refiere al caso de Ada Jukes, nacida en 1740, y que estaría en el origen de un árbol genealógico trágico: entre sus descendentes aparecen 64 débiles mentales; 174 perversos sexuales, 196 ilegítimos, 77 criminosos y asesinos y, representando un estigma moral, 147 pobres. El cuadro se inicia con la siguiente afirmación: "su esterilización tendría costado 150 dólares, y concluye con la siguiente pregunta: ¿Dejaremos que las Adas Jukes de hoy continúen multiplicando la pobreza y la criminalidad?" (Gouyon, Henry, 1997, p.93). De acuerdo a Philippe Reilly (1987, p.153):

El acontecimiento más importante que precedió al surgimiento de los programas de esterilización fue probablemente la publicación del estudio de Richard Dugdale (1877) sobre los Jukes; Una familia de Nueva York con una propensión a los hospicios, las tabernas, los burdeles, y los cárceles. El 'Jukes' engendró un nuevo campo en sociología: estudios de campo extensos de familias degeneradas, un campo que alcanzó su apogeo en la Oficina de Registro Eugenésico (ERO). De 1910 a 1914 aparecieron más de 120 
artículos sobre eugenesia en revistas, un volumen de impresión que lo convierte en uno de los temas favoritos de la nación.

El caso de la familia Jukes es referenciado por Kehl en Lições de eugenia. En la nona lección, titulada "Consideraciones generales y estadísticas sobre las degeneraciones de orden tóxica", dedicará un extenso párrafo a la familia Jukes, afirmando que la esterilización podría haber evitado la propagación de una prole degenerada por el alcoholismo, que cuenta entre sus miembros diversos enfermos mentales y criminales (Kehl, 1929, p.150). Otras genealogías son presentadas en este libro, fundamentalmente provenientes de casos relatados en EEUU, por Davenport (1911) y de Alemania, por Ernst Rudin $(1937,1938)$. Todas las genealogías presentadas tienen el objetivo de confirmar la importancia de crear un espacio público para la organización de las genealogías en Brasil, siguiendo el modelo de la Oficina de Registro Eugenésico (ERO) de EEUU. Para Kehl (1929, p.32-33),

Llegará un día en el cual el Estado asumirá el control del 'creced y procreaos', comenzando por organizar la genealogía de toda la gente ... De ese modo será creado un Registro Genealógico Oficial destinado a destacar la nobleza de la elite ... Los individuos considerados ineptos a la procreación tendrán apenas derecho a los placeres sexuales cuando hayan sido previamente esterilizados.

Cinco años más tarde, en 1934, en el Arquivo Brasileiro de Neuriatria y Psiquiatria, el siquiatra Cunha Lopes, publica un artículo denominado "Da esterilização em psiquiatria", nuevamente es en las genealogías y los estigmas donde encontrará los argumentos para defender la esterilización de enfermos mentales, oligofrénicos, personas con sordomudez y ceguera congénita. Cunha Lopes presenta estadísticas elaboradas en Alemania por autores como Ernst Rudin y Fritz Lenz, argumentando, como Kehl, que es urgente la realización de genealogías con fines de esterilización, destacando las proporciones en las que son afectados familiares de personas que sufren esquizofrenia, maniacos depresivos, oligofrénicos y epilépticos en las siguientes relaciones familiares: hermanos, hijos, primos, sobrinos, nietos, sobrinos nietos y bisnietos, comparativamente con la población general. Una suma, en fin, de estadísticas extrañas y arbitrarias, donde no solo aparecen referencias al grupo patológico estudiado sino a la posibilidad de que pueda ocurrir cualquier otro tipo de patología. Cunha Lopes (1934, p.261) concluye este artículo felicitándose por el trabajo realizado para la reciente creación de la sección de Genealogia e Estatística da Assistência a Psicopatas do Distrito Federal, diciendo que estas genealogías "futuramente darán una obra grandiosa, de vastísimo alcance en la defensa médico-social del país".

Tal como afirman Robert Wegner e Vanderlei de Souza (2013), el discurso de Renato Kehl comenzará a radicalizarse y a abandonar las posiciones inicialmente próximas a las intervenciones higiénicas y sociales, para asumir un discurso radicalmente conservador y racista. De acuerdo a estos autores, el cambio de posición teórica y política de Kehl ocurre cuando es designado director de la Indústria Química e Farmacéutica Bayer do Brasil, con sede en Rio de Janeiro, al final de la década de 1920.

Un año después de haber asumido la dirección de la casa Bayer de Brasil, Renato Kehl fue invitado por la multinacional alemana para realizar un viaje de cinco meses por el norte de Europa, especialmente por Alemania. En ese viaje, visitó varias universidades 
e institutos de antropología y eugenesia alemanas y de otros países del norte europeo ... En sus correspondencias particulares e institucionales es posible percibir relaciones intelectuales frecuentes con médicos, psiquiatras, biólogos, antropólogos y eugenistas, muchos de ellos directores de institutos, asociaciones, revistas y periódicos relacionados con el movimiento eugenista europeo (Wegner, Souza, 2013, p.5).

En 1924, el primer número de los Arquivos Brasileiros de Higiene Mental publica un artículo de Renato Kehl denominado: "A esterilização dos grandes degenerados e criminosos", luego reeditado como capítulo en el libro Lições de eugenia (1929). De acuerdo al relato de Ernani Lopez (1933), el calificativo de "grandes" utilizado en el título, fue una concesión realizada por Kehl, a pedido de un grupo de siquiatras, para evitar críticas y no herir susceptibilidades de los colegas.

En la misma revista, el siquiatra Alberto Farani (1931) publica un texto denominado "Como evitar as proles degeneradas". Allí afirma: "Los degenerados mentales por todas las estadísticas aumentan de modo constante exigiendo de la sociedad medidas de protección. Aquí no se trata de medios de anticoncepción sino de impedimento definitivo. Esto solo puede ser obtenido por esterilización y restricción matrimonial" (p.174).

Este debate se había iniciado años antes, en el primer Congreso Brasilero de Eugenesia, llegando a un consenso establecido en el acta de la reunión de 7 de junio de 1929. Allí leemos: "Entran en votación y son aprobadas las siguientes conclusiones: (1) Son benignas del punto de vista quirúrgico, la vasectomía y la laqueadura tubaria, (2) Es legítima la esterilización de los degenerados de acuerdo a las indicaciones de los neuro-psiquiatras" (Carneiro, 1929, p.38).

Ante la imposibilidad de lograr la aprobación de una ley como esa, cuatro años más tarde, el siquiatra Ernani Lopes (1933) publica un artículo en los Arquivos Brasileiros de Higiene Mental, denominado: "A alta tardia dos heredo-psicopatas por motivos de ordem eugénica". Ese artículo pone en evidencia las resistencias que la Sociedad Brasilera de Eugenesia encontró para la aceptación de los principios de esterilización de los degenerados, aprobada en el congreso de 1929. Inconformado ante esta imposibilidad, Ernani Lopes (1933, p.280) propone otra alternativa: el secuestro de los enfermos mentales en hospitales psiquiátricos, afirmando que: "No debemos desconocer la realidad. Confesemos que una ley permitiendo la esterilización eugénica, despierta aún gran oposición en nuestro medio. Encaremos por lo tanto, otro aspecto de la restricción eugénica: la retirada de los heredo-psicópatas del medio social por motivos de orden eugénica, esto es, para evitar que ellos se procreen".

Un año más tarde, los Arquivos Brasileiros de Neuriatria e Psiquiatria publican un artículo del siquiatra Cunha Lopes (1934) denominado "A esterilização em psiquiatria". Este texto se refiere a la esterilización de los degenerados hereditarios enunciando argumentos políticos (Nalli, jul.-dez. 2005) próximos a las ideas defendidas por los siquiatras y eugenistas alemanes. En el texto mencionado, Cunha Lopes (1934, p.257) defiende la esterilización involuntaria de enfermos psiquiátricos afirmando que "es la ley alemana sancionada en 14 de julio de 1933, la que satisface la más amplia aceptación de la eugenesia de la que se tenga noticia. Representa un trabajo persistente realizado metódicamente en todo el Reich desde la gran guerra hasta la osada ascensión de Hitler". El autor concluye su trabajo defendiendo una propuesta de legislación que, felizmente, nunca fue aprobada. El parágrafo $1^{\circ}$ afirma: 
"El enfermo hereditario puede, por intervención quirúrgica ser esterilizado si, de acuerdo al conocimiento de la ciencia médica puede esperarse con probabilidad, que su descendencia pueda sufrir daños hereditarios psíquicos" (Lopes, 1934, p.257).

Vemos que la asociación entre degeneración y eugenesia se mantuvo inalterada, en los argumentos de algunos siquiatras brasileños, vinculados al Boletim de Eugenia, siendo reforzada cuando se persiguen objetivos como la esterilización, el secuestro o el aislamiento de los enfermos mentales.

\section{Consideraciones finales}

Analizamos hasta aquí dos modelos deterministas (Rose, 2001) de explicación y de intervención sobre las poblaciones consideradas anormales, que pudieron coexistir en las primeras décadas del siglo XX en diversos países. Un modelo representado por Magnan y la teoría de la degeneración y otro representado por Galton y los defensores de la eugenesia. Dos modelos explicativos que encuentran un punto de convergencia en el "mito de la herencia mórbida y de la normalidad hereditaria".

El determinismo hereditario de Magnan se pone en evidencia cuando afirma: "El degenerado es un enfermo en el origen ad ovo. Se presenta con una tara constitucional que lo acompaña toda la vida de la cuna a la tumba" (Magnan, Legrain, 1895, p.93). De igual modo, el determinismo aparece en Galton (1930, p.2) cuando leemos que "las historias de vida de nuestros ancestrales son profecías de nuestro propio futuro". Estos modelos no suponen simplemente dos marcos explicativos deterministas, sino que inducen y producen estrategias efectivas de intervención. Se trata, en fin, de dos dispositivos biopolíticos, centrados en herencia mórbida, profundamente conservadores y promotores de estrategias autoritarias de intervención y control social. Estos dispositivos son diferentes en la medida que la teoría eugenésica de Galton, sus ideas sobre herencia mórbida y desvíos estadísticos fueron construidas con absoluta independencia y sin ninguna referencia a la teoría de la degeneración de Morel o Magnan.

Sin embargo, como intenté mostrar aquí, la asociación entre degeneración y eugenesia se repite incansablemente en el discurso de los eugenistas como Rudin o Renato Kehl. Esta diferencia aparece ya en las primeras páginas del libro de Kehl, Lições de eugenia, cuando enuncia dos definiciones de eugenesia. La primera es la definición oficial, elaborada en 1904 por la Sociedad Eugenésica de Londres: "El termino eugenesia debe ser definido como el estudio de los factores que, bajo control social, pueden mejorar o perjudicar las cualidades raciales de las generaciones futuras, sea física o mentalmente" (Kehl, 1929, p.17). La segunda definición, elaborada por Renato Kehl (1929, p.17), afirma que "el programa de la eugenesia puede resumirse en: favorecer la estabilización de las cualidades hereditarias óptimas e impedir la transmisión de caracteres degenerativos transmisibles hereditariamente, organizando la sociedad humana contra los factores de degeneración". Entre una definición y otra existe una diferencia evidente, el lugar central reservado por Kehl para la noción de degeneración.

Como ya fue dicho, degeneración y eugenesia se identifican por la importancia dada a la idea de herencia mórbida. Vimos, sin embargo, que estas teorías parten de marcos 
teóricos diferentes para explicar la herencia. Tal como lo explica el texto de José Leme Lopes, publicado en 1945, con el nombre "O problema da herança em psiquiatria":

El contraste entre la siquiatría del siglo pasado y el actual es llamativo, al estudiar el problema de la herencia de las enfermedades mentales. Todo el siglo XIX está marcado por las ideas de Morel, relativas a la degeneración, a la deviation maladive, de la cual las psicopatías serían un resultado. Magnan y Lombroso, después de Morel, volvieron a insistir en la herencia progresiva, polimorfa y agravada, buscando explicar por ella casi toda la patología del espíritu (Lopes, 1945, p.68).

El autor afirma que fue solamente con el redescubrimiento de los trabajos de Mendel que la investigación heredológica, en el campo de la psiquiatría, se transformó en un instrumento seguro. Para luego afirmar que "fue Rudin quien, en 1911, inauguró una nueva era para la investigación genotípica en psiquiatría, basada en el mendelismo" (Lopes, 1945, p.69).

Algo semejante ocurre con la identificación de estigmas. Autores como Apert (1919) ya habían mostraron la limitación y las dificultades existentes en el reconocimiento de una lista infinita de estigmas de degeneración, y su escaza operatividad para la identificación de factores considerados disgénicos. Sin embargo, la incansable búsqueda por estigmas de degeneración reaparece una y otra vez, no solo en los textos de Renato Kehl, sino también en diferentes artículos publicados en el Boletim de Eugenia.

Las mismas diferencias se repiten cuando nos referimos a las genealogías y a la búsqueda de árboles genealógicos. Aun cuando el conocimiento de las enfermedades de los ascendientes y descendientes es considerado por Magnan y los degeneracionistas como una información esencial para definir y clasificar una patología psiquiátrica, en el caso de los eugenistas la formación de historias de familia y arboles genealógicas aparece como siendo una tarea central y esencial del programa. En el artículo publicado por Galton (1930, p.2) en el Boletim de Eugenia, denominado "A história da família e genealogia", dirá que "parece no haber tiempo que perder para estimular y organizar la compilación de datos para la formación de historias familiares". Estas historias familiares o genealogías no son idénticas a las realizadas por los degeneracionistas. Estos últimos coleccionaban casos e historias familiares de sicopatología, observando cómo se repetían o agravaban las enfermedades en las sucesivas generaciones y desconsiderando la distinción entre "enfermedades recesivas y dominantes". De acuerdo a Lemes Lopes (1945, p.69): "El gran mérito de Rudin fue mostrar los errores de los siquiatras al dedicarse al estudio de familias con fuertes taras, a la recolección y publicación de árboles genealógicos cargados de sicopatías. El interés debe dirigirse al mismo tiempo para las familias con poca o ninguna tara".

Todo parece indicar que el concepto de degeneración poco puede aportar a los estudios e intervenciones eugénicas. Existen diferentes formas de entender la herencia, los estigmas y los árboles genealógicos, además de existir enormes diferencias en las estrategias de intervención como la esterilización o el alta tardía por motivos eugenésicos. Siendo así, no podemos dejar de interrogarnos por la persistencia de esta asociación en autores como Renato Kehl o Ernst Rudin $(1937,1938)$. 
Quizás sea preciso encontrar la explicación en otra parte, ya no en la idea de herencia, sino en las posibilidades abiertas por esa categoría siquiátrica amplia que se define con el nombre de los heredo-degenerados. Es así que Franco da Rocha (2008, p.151) define, en 1904, a ese agrupamiento patológico: "Hay individuos, y se cuentan por legiones, que no son declaradamente locos ni de mentalidad perfectamente normal: son los degenerados que, gradualmente, sin línea bien definida, establecen la transición entre el loco y el sano de espíritu".

Lo que la teoría de la degeneración permite es considerar un número indefinido de comportamientos, características y actitudes como desequilibrios o trastornos mentales. De modo que siempre será posible adicionar una nueva patología a la extensa lista de esas degeneraciones mentales. En la medida en que no existe una línea de demarcación definida entre lo normal y lo patológico, habrá "legiones de individuos" que no son considerados ni locos, ni normales, los degenerados.

La degeneración es definida por Kehl (1929) como un desvío, más o menos pronunciado, del individuo sano, fundamentalmente por factores congénitos y hereditarios, sin descartar factores de otra índole. Se trata de una definición ambigua e imprecisa, pero es esa imprecisión la que le otorga un poder infinito en la medida en que puede ser incesantemente ampliada. Esa elasticidad permite afirmar que "gran parte de nuestros semejantes es víctima de enfermedad o de achaques o de taras o de degeneraciones" (p.49). Kehl propone una suerte de juego, nos invita a que observemos diez personas próximas, para que rápidamente podamos comprobar que aun entre las personas más cercanas podremos identificar degeneraciones y enfermedades de mayor o menor gravedad (p.50).

Esa capacidad de ampliar indefinidamente el campo de lo patológico fue destacada por diversos críticos de la degeneración, como Gilbert Ballet (citado en Apert, 1919, p.262):

La palabra degeneración del punto de vista etiológico, tiene una significación vaga y variable, y lejos de aportar al lenguaje la claridad de un término neto y preciso, tiene la función de llevar confusión a los espíritus... Yo me he servido como todo el mundo de la palabra degeneración, pero más veo y más me convenzo de que la razón que le ha asegurado el suceso, esto es su vaguedad y la comodidad de su uso, es precisamente lo que debe ser rechazado.

La degeneración presenta un marco vago y ambiguo de referencia, que permite ampliar indefinida la injerencia de la siquiatría sobre los problemas humanos (Foucault, 1999). Atribuyendo un significado exagerado a los menores desvíos y anomalías, siendo difícil encontrar un individuo que está libre de todo estigma de degeneración.

Así, si degeneración y eugenesia pudieron complementarse y asociarse sin dificultad, a pesar de las diferencias señaladas, es porque allí es donde los críticos de la degeneración ven una limitación, Kehl encuentra su riqueza y operatividad. La elasticidad de la noción de degeneración contribuye a los sueños totalitarios de autores como Kehl o Rudin. La ambigüedad y vaguedad de la degeneración parecería legitimar que las estrategias eugénicas como la esterilización, la interdicción de matrimonios, la internación siquiátrica con fines eugenésicos, pueda extendersede forma ilimitada a ese mundo confuso e indefinido,mundo situado entre la completa locura y la completa normalidad. Y es en ese marco que podemos 
situar esta afirmación de Kehl (1929, p.50; énfasis en el original): "Somos de la opinión que la esterilización es indicada en casos especiales de enfermedad y de 'miseria', que debe ser aplicada compulsivamente a ciertos criminales y en casos de degeneración somato-síquica. Debe ser considerada como un inestimable proceso eugenésico".

Nos referimos ya a las deudas de Kehl con los siquiatras y eugenistas alemanes (Rolim, Sá, 2013), quienes llevaron la eugenesia y la degeneración hasta su límite extremo. La degeneración con su significado vago y variable, incapaz de aportar claridad al campo de la siquiatría, pudo aliarse perfectamente al discurso eugenista, en la medida que justamente por su vaguedad, extensión y comodidad permitía ampliar indefinidamente su campo de intervención. Esa vaguedad le permitió a Kehl y a sus seguidores, como le permitió a Ernst Rudin y a Davenport, llevar hasta el límite la ambición totalitaria de controlar e intervenir en la vida de los individuos, particularmente en el mundo de todos aquellos que, a sus ojos, eran considerados anormales e indeseables.

\section{NOTA}

${ }^{1}$ En esta y en las demas citas literales de textos publicados en otros idiomas la traducción es libre y fue proporcionada por la autora.

\section{REFERENCIAS}

APERT, Eugene.

L'Heredité morbide. Paris: Flammarion. 1919.

BOARINI, Maria Lucia.

Higiene mental: ideias que atravessaram o século XX. Maringá: Editora da UEM. 2012.

CAROL, Anne.

Histoire de l'eugenisme en France. Paris: Seuil. 1995.

CARNEIRO, Levi.

Ata da sétima reunião, 7 de julho de 1929. In: Congresso Brasileiro de Eugenia, 1., 1929, Rio de Janeiro. Atas e trabalhos. Rio de Janeiro: [s.n.]. p.37. 1929.

COFFIN, Jean-Christophe.

La transmission de la folie, 1850-1914. Paris:

Harmattan. 2003.

DAVENPORT, Charles.

Heredity in relation to eugenics. New York: Henry Holt. 1911.

DORON, Claude-Olivier.

L’homme altéré: races e dégénérescence. Paris: Champ Vallon. 2016.

DUGDALE, Richard.

The Jukes: a study in crime, pauperism, diseases, and heredity. New York: Putnam's Sons. 1877.
FARANI, Alberto.

Como evitar as proles degeneradas. Arquivos Brasileiros de Higiene Mental, ano 4, n.3, p.169179. 1931.

FONSECA, Joaquim Moreira da.

Casamento e eugenia. In: Congresso Brasileiro de Eugenia, 1., 1929, Rio de Janeiro. Atas e trabalhos. Rio de Janeiro: [s.n.]. p.299-304. 1929.

FOUCAULT, Michel.

Les anormaux. Paris: Seuil. 1999.

GALTON, Francis.

Herencia y eugenesia. Madrid: Alianza. 1988.

GALTON, Francis.

A historia da familia e genealogia. Boletim de Eugenia, ano 2, n.16, p.1. 1930.

GOUYON, Jean Pierre; HENRY, Jaques. Les avatars du gene. Paris: Belin. 1997.

HUERTAS, Rafael.

Otra historia para otra psiquiatría. Madrid: Xoroi. 2017.

KEHL, Renato.

Lições de eugenia. Rio de Janeiro: Francisco Alves. 1929.

KEHL, Renato.

A esterilização dos grandes degenerados e criminosos. Arquivos Brasileiros de Higiene Mental, ano 1, n.2, p.69-74. 1925. 
KRAEPELIN, Emil.

On the question of degeneration. History of

Psychiatry, v.18, p.398-404. 2007.

KRAEPELIN, Emil.

One hundred years of psychiatry. New York:

Philosophical Library. 1917.

KRAEPELIN, Emil.

Clinical psychiatry: a text book for students and physicians. New York: Macmillan. 1907.

KRAFFT-EBING, Richard.

Text book of insanity. Philadelphia: Davis. 1903.

LOPES, Ernani.

A alta tardia dos heredo-psicopatas por motivos de ordem eugénica. Arquivos Brasileiros de Higiene Mental, ano 6, n.4, p.277-289. 1933.

LOPES, Ignácio da Cunha.

Da esterilização em psiquiatria. Arquivos

Brasileiros de Neuriatria e Psiquiatria, ano 17, n.4, p.252-261. 1934.

LOPES, José Leme.

O problema da herança em psiquiatria. Arquivos de Neuro-psiquiatria, v.3, n.1, p.68-77. 1945.

MAGNAN, Valentin.

Recherches sur les centres nerveux: alcoolisme, folie des héréditaires dégénérés. Paris: Masson. 1893.

MAGNAN, Valentin; LEGRAIN, Paul.

Les degénérés: état mental et syndromes épisodiques. Paris: Rueff. 1895.

MINKOWSKI, Eugene.

Le schematisme de la loi de esterilisation obligatoire en regard du probleme de l'heredité e de celui de l'alienation mentale. Annales Médico-psycologiques, n.1, p.481-485. 1938.

MINKOWSKI, Eugene.

Discussion sur les condition et le rol de l'eugenique dans prophylaxie des maladies mentales. Annales Médico-psycologiques, n.2, p.371-372. 1937.

MIRANDA, Marisa, VALLEJO, Gustavo (Ed.). Darwinismo social y eugenesia en el mundo latino. Buenos Aires: Siglo XXI. 2005.

MOREL, Benedict August.

Rapport fait à la Société Medico-psicologique sur le Traité de Dégénérescence. Annales Médicopsicologiques, n.3, p.4. 1859.

MOREL, Benedict August.

Traité des dégénérescences physiques, intellectuelles et morales de l'espèce humaine et des causes qui produisent ces varieties maladives. Paris: Baillière. 1857.
NALLI, Marcos.

Antropologia e racismo no discurso eugênico de Renato Kehl. Teoria e Pesquisa, n.47, p.119-156.

jul.-dez. 2005.

PICHOT, André.

L'eugénisme. Paris: Hatler. 1995.

PIETTA, Gerson; CARVALHO, Leonardo.

Um diagnóstico para os acervos: a eugenia nas publicações de Renato Kehl e João Candido Ferreira, 1920- 1930. Patrimônio e Memória, v.12, n.2, p.4-26. 2016.

REILLY, Philip.

Involuntary sterilization in the United States: a surgical solution. The Quarterly Review of Biology, v.62, n.2, p.153-170. 1987.

RICHET, Charles.

La selection humaine. Paris: Baillière. 1922.

ROCHA, Francisco Franco da.

Esboço de psiquiatria forense [1904]. Revista

Latino-americana de Psicopatologia Fundamental, v.11, n.1, p.151-165. 2008.

ROLIM, Marlon Silva; SÁ, Magali Romero. A política de difusão do germanismo por intermédio dos periódicos da Bayer: a revista terapêutica e o farmacêutico brasileiro. História, Ciências, Saúde - Manguinhos, v.20, n.1, p.159179. 2013.

ROQUETTE-PINTO, Edgard.

Sessão inaugural de 1 de julho. In: $1^{\circ}$ Congresso Brasileiro de Eugenia, 1929, Rio de Janeiro. Atas e trabalhos. Rio de Janeiro: [s.n.]. p.11-12. 1929.

ROSE, Steven.

Trayectorias de vida: biología, libertad y determinismo. México: Granica. 2001.

RUDIN, Ernest.

La profilaxie du mariage. Annales Médicopsycologiques, n.2, p.628-632. 1938.

RUDIN, Ernest.

Les condition et le rol de l'eugenique dans prophylaxie des maladies mentales. Annales Médico-psycologiques, n.2, p.370. 1937.

SÉGLAS, Jules.

Leçons cliniques sur les maladies mentales et nerveuses. Paris: Libraires de la Faculté de Médecine. 1895.

SERPA, Octavio.

Dégénérescence. In: Andrieu, Bernard (Org.).

Dictionnaire du corps en sciences humaines et sociales. Paris: CNRS. 2006.

SOUZA, Vanderlei Sebastião de. A eugenia brasileira e suas conexões internacionais: uma análise a partir das 
controvérsias entre Renato Kehl e Edgard Roquette-Pinto, 1920-1930. História, Ciências, Saúde-Manguinhos, v.23, supl., p.93-110. 2016.

SOUZA, Vanderlei Sebastião de.

A política biológica como projeto: a "eugenia negativa" e a construção da nacionalidade na trajetória de Renato Kehl, 1917-1932. Dissertação (Mestrado em História das Ciências e da Saúde) - Casa de Oswaldo Cruz, Fiocruz, Rio de Janeiro. 2006a.

SOUZA, Vanderlei Sebastião de.

Em nome da raça: a propaganda eugênica e as ideias de Renato Kehl nos anos 1910 e 1920. Revista de História Regional, v.11, n.2, p.29-70. 2006b.
SOUZA, Vanderlei Sebastião de.

A política biológica como projeto: a "eugenia negativa" e a construção da nacionalidade na trajetória de Renato Kehl, 1917-1932. História, Ciências, Saúde - Manguinhos, v.13, n.4, p.10621063. 2006c.

STEPAN, Nancy.

A hora da eugenia: raça, gênero e nação na América Latina. Rio de Janeiro: Editora Fiocruz. 2005.

WEGNER, Robert; SOUZA, Vanderlei Sebastião de. Eugenia "negativa", psiquiatria e catolicismo: embates em torno da esterilização eugênica no Brasil. História, Ciências, Saúde - Manguinhos, v.20, n.1, p.263-288. 2013. 